\title{
Improvement of Cut Quality in Rapid-Cutting of Glass Method via Femtosecond Laser Filamentation
}

\author{
Simas BUTKUS ${ }^{* 1}$, Domas PAIPULAS ${ }^{* 1}$, Dalia KAŠKELYTE் ${ }^{* 1}$, Eugenijus GAIŽAUSKAS ${ }^{* 1}$ and \\ Valdas SIRUTKAITIS ${ }^{*}$ \\ ${ }^{1 *}$ Vilnius University, Faculty of Physics, Department of Quantum Electronics, Laser Research Center, Sauletekio av. 10, \\ Vilnius 10223, \\ E-mail:Simas.Butkus@ff.vu.lt
}

\begin{abstract}
Microfabrication of transparent materials using femtosecond laser pulses has showed good potential towards industrial application. Maintaining pulse energies above the critical self-focusing limit produced filaments that were used for micromachining purposes. This article demonstrates a fabrication technique using femtosecond filaments generated in water which is in contact with the transparent sample, in addition, given a slight modification to this setup, the quality of the micro processed samples can be increased dramatically. These fabricated holes have a tapper angle close to zero; moreover, the fabrication time is of the order of tens of seconds.
\end{abstract}

DOI: $10.2961 / \mathrm{jlmn} .2015 .01 .0012$

Keywords: Femtosecond pulse micromachining, filament processing, ablation, glass cutting

\section{Introduction}

Femtosecond laser pulses (high intensity) are a convenient means for various material micromaching. Due to their unique properties the nonlinearities of the medium are invoked which, in turn, causes nonlinear absorption or self-actions of the beam, i.e. due to the high intensity of femtosecond pulses the energy of the pulse can be absorbed even if the photon energy is less than the bandgap of the material, therefore these laser systems present the ability to fabricate virtually any type of material [1].

Though femtosecond lasers show superiority over other types of lasers, these systems are considerably more expensive and implementation of such systems may be more complicated. In addition, certain materials e.g. brittle glasses or crystalline materials can not withstand pressure or temperature gradient formation therefore high average optical power use may often be restricted yet timeefficiency needs demand high power use for precise, rapid fabrication. Such paradoxical situations are solved through parameter optimisation and tuning the process to fit the desired tasks. A number of articles have been published on femtosecond laser microfabrication of transparent materials using different approaches: material removal through direct laser ablation (front side and water assisted back side) [2-6], ablation enhanced by spatial and temporal focusing [7-9] and others. Alternative fabrication techniques are also present: chemical etching of laser induced bulk modifications [10-15], glass dicing techniques [16, 17]. It can be noted, that the above presented techniques are capable of performing high quality fabrication, although implementation of such techniques typically requires high numerical aperture (NA) focusing conditions, precise motion translations stages or additional components leading to applications only for specific goals. In addition, time-efficiency and fabrication quality suffer when slight fluctuations in parameter values are present due to greater sensitivity of such systems [18]. Ultimately, in order to achieve greater fabrication quality, one must sacrifice timeefficiency or vice versa, as in the case of conventional (mechanical) machining methods [19].

In the case of material removal via ablation, the imperfections that occur during fabrication are due to several reasons. Firstly, heat build up in the impinged regions may occur during multiple pulse interaction which leads to cracking and chipping [20]. Secondly, during fabrication grooves or holes are formed and their shape changes after a number of pulses impinge on the surface, i.e. the grooves or holes become deeper as more pulses impinge a surface region. The electric field of the impinging subsequent pulses on the groove diffracts, refracts, reflects and forms interference patterns with its self on the groove walls. Due to these interference patterns the intensity distribution of the pulse modifies, which in turn results in $\mu \mathrm{m}$ scale cracks, conicity of the groove and regions with induced stress that ultimately lead to chipping [21]. Therefore it can be noted, that the imperfections that occur during fabrication are due to the fundamental properties of the light pulses and these flaws can not be easily overcome.

In this work we present transparent material cutting and drilling results achieved with a fabrication setup that employs filamentation. By applying a water layer on top of the glass samples and using a low NA objective, filaments can be created near the surface of the glass. Filaments are formed within the water layer and are used for micromachining purposes. Experimental results using this this particular micromachining approach will not be presented here in great detail, however they can be found in [22] where, in addition, numerical simulations of filament formation along with extensive parametric investigations are presented. The used approach is adventageous in various ways e.g. $\mathrm{z}$ axis translation is not required while several $\mathrm{mm}$ deep structures can be fabricated, additional cooling is provided due to the added water layer which 
prevents temperature and pressure gradient formation, large scanning rates are achievable and etc.

At the core of the filament, strong electron plasma generation and electron relaxation causes ablation at the surface of the glass samples. As most fabrication techniques this one also generates imperfections in the processed samples. In addition, we present a technique that contains these defected regions thus dramatically increasing the quality of the processed samples while the reduction in fabrication rate is minimal. In this $2 \mathrm{D}$ hole cutting and drilling technique filamentation is favourable due to its surplus focusing abilities, hence complex $\mathrm{Z}$ axis positioning systems and sharp focusing optics can be eliminated. Moreover, it enables the use of galvanometric scanners in combination with $\mathrm{f}$ - theta lenses producing large scanning fields and scanning rates of the order of $\mathrm{m} / \mathrm{s}$ or greater. The complexity of this system was brought to a minimum bearing in mind that non scientific personnel might be the end-user of such a system.

\section{Experimental setup}

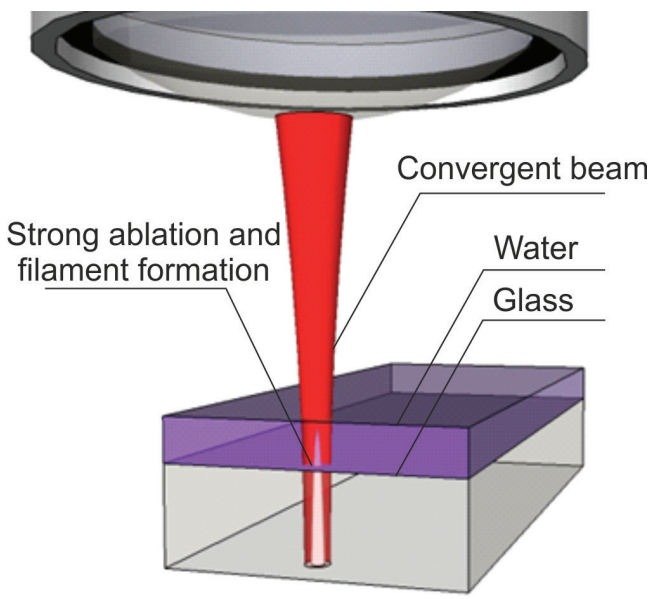

Fig. 1 Focusing conditions of the microfabrication system

The experiments were carried out using the $\mathrm{Yb}: \mathrm{KGW}$ femtosecond laser system Pharos ${ }^{\circledR}$ (Light Conversion Ltd). A two axis galvanometric scanner (ScanLab Inc.) that was controlled by fabrication software SCA (Altechna Ltd). The beam was focused using an F- theta lens $(\mathrm{f}=100 \mathrm{~mm}$ ) inside of the glass sample which was covered with a water layer $(0.5-1 \mathrm{~mm}$ thick $)$ such that filament formation occurs near the interface of the transparent materials. Fig. 1 shows a schematic drawing of the focusing conditions. Drilling and cutting was carried out in soda-lime glass (thickness $1 \mathrm{~mm}$ ) samples. The water layer was formed by submerging the glass samples in a reservoir (not shown here). The thickness of this film was measured by shining a beam from a green laser pointer onto the surface of the water film and measuring how the position of the reflected beam (from the water layer) changes on a screen when more or less water is poured in the reservoir. It was found that the thickness can be determined to an accuracy of about 50 $\mu \mathrm{m}$. Typical writing parameters were chosen as follows: average pulse power - $5 \mathrm{~W}$, pulse duration - $280 \mathrm{fs}$, repetition rate - $25 \mathrm{kHz}$, pulse energy - $200 \mu \mathrm{J}$, wavelength - $1030 \mathrm{~nm}$, focal spot diameter at the focus $55 \mu \mathrm{m}$.

Using the setup presented in Fig. 1 cutting of round shape holes and complex shape objects was carried out, in addition, using a slightly modified setup (will be presented shortly) similar experiments were carried out yielding better results in term of quality. Both of the techniques were compared and discussed in the forthcoming sections.

\section{Results and discussion \\ 3.1 Cutting and drilling of transparent materials}

The strategy for transparent material drilling presented here is employment of direct ablation from the top surface of the sample. Ablation in this case is induced by the intense laser pulse generated filament. In this configuration no post-processing is necessary (holes are drilled on the spot) and the range of the materials that can be processed using this method is wide due to only a mild dependence on the chemical composition of the material, i.e. virtually any type of material (glasses, crystals, metals and etc.) can be processed using this method. The utilization of filamentation for micromachining purposes is beneficious due to a few reasons. First, when the filament forms (the Gaussian beam is transformed to a Gauss-Bessel beam) the intensity distribution along the $\mathrm{z}$ direction is altered in a way that transcends diffraction, i.e. the intensity along the $z$ direction stays constant for a far longer propagation length relative to what it would be just with the focusing lens with no self-actions present. This results in decreased sensitivity in $\mathrm{z}$ axis positioning and removes the need for translation while fabricating. Second, the physical dimensions (can be as low as $10 \mu \mathrm{m}$ [23]) of the filement's core are far less compared to conventional focusing with the same lens, in addition, the intensity distribution is more uniform. Better intensity uniformity produces stepper edges and produces greater flatness in the impinged zone [24]. Overall, these properties of the filamented beam produce greater fabrication throughput with better quality and reduced sensitivity to parameter fluctuations.

Using the setup presented in Fig. 1 cutting and drilling of transparent materials was carried out. The parameters listed in section 2 were chosen such that would yield maximum average power $(5 \mathrm{~W})$ and maximum pulse energy $(200 \mu \mathrm{J})$ in this specific laser system. The water layer on the top of the sample acts as a buffer layer where filamentation occurs. The thickness can be controlled by dosing the quantity of water poured in the reservoir (however in the experiments presented here the thickness was fixed to $0.5 \mathrm{~mm}$ ). In addition, this layer helps reduce thermal effects and remove debris from the processed surface due to cavitation bubble formation and collapse [25]. It is worth pointing out that the water film can additionally increase the ablation rate through a process known as laser-induced plasma confinement. The plasma that is being formed at the glass sample's front surface is being confined via the water layer, which results in better plasma-target coupling $[26,27]$ therefore the interaction of 


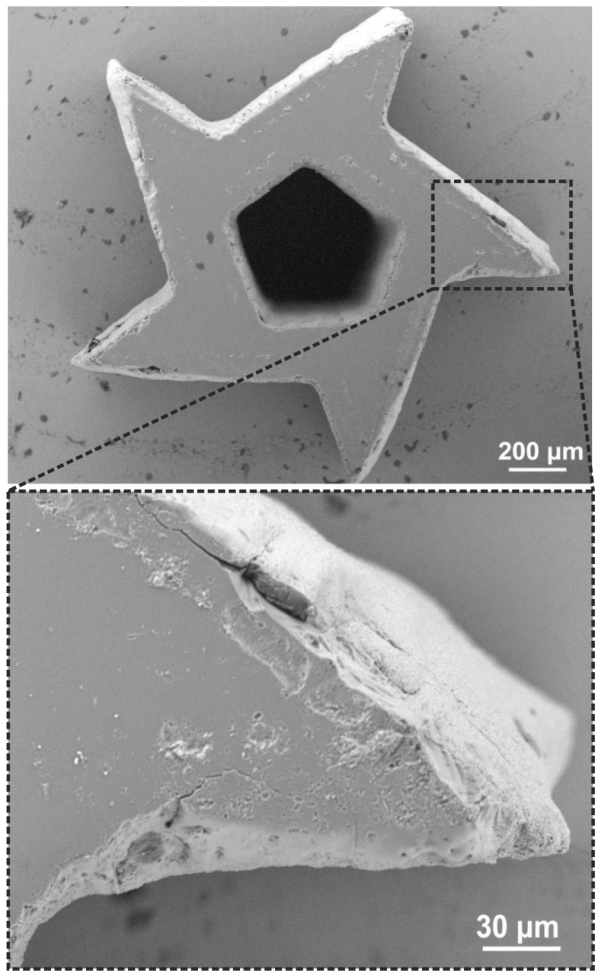

Fig. 2 Complex shape object fabricated in $1 \mathrm{~mm}$ thick sodalime glass in approximately 3 minutes. $5 \mathrm{~W}, 200 \mu \mathrm{J}, 25 \mathrm{kHz}$, $280 \mathrm{fs}, 100 \mathrm{~mm} / \mathrm{s}$.

the plasma with the glass is being prolonged which results in more efficient ablation.

In order to carry out the most efficient fabrication using this particular micromachining system other parameters such as: scanning speed (subsequent pulse overlap), focal position (position of where the intense filament forms), water layer thickness have to be fine tuned. As mentioned, parameter optimisation results will not be presented here. The parameters that produce the most efficient fabrication using this setup are as follows: scanning speed $-100-150$ $\mathrm{mm} / \mathrm{s}$, focal position (position of lens geometrical focus) 1.5 - $2 \mathrm{~mm}$ beneath the surface of the glass sample, water film thickness $-0.5 \mathrm{~mm}$, i.e. using the above mentioned parameters the glass sample is cut through in the least amount of time and the quality of the cuts is highest.

\subsection{Improving cut quality by covering the glass sample with an additional glass layer}

Using the technique desribed in section 2 drilling of holes and cutting of complex shape objects is achievable. Holes having $2 \mathrm{~mm}$ in diameter can be cut in approximately 40 seconds in $1 \mathrm{~mm}$ thick soda-lime glass. An example of complex shape fabrication is presented in Fig. 2. One can notice the imperfections at the bottom part of the figure. These imperfections can not be eliminated by changing the processing parameters. As stated these imperfections are due to the fundamental properties of the light beam's interaction with the surface of the sample. Therefore a different approach has to be chosen in order to improve the quality of the processed samples.

By putting a secondary glass layer on top of the base layer (shown as primary in Fig. 3) the quality of the cuts can be increased dramatically. It is a well known concept

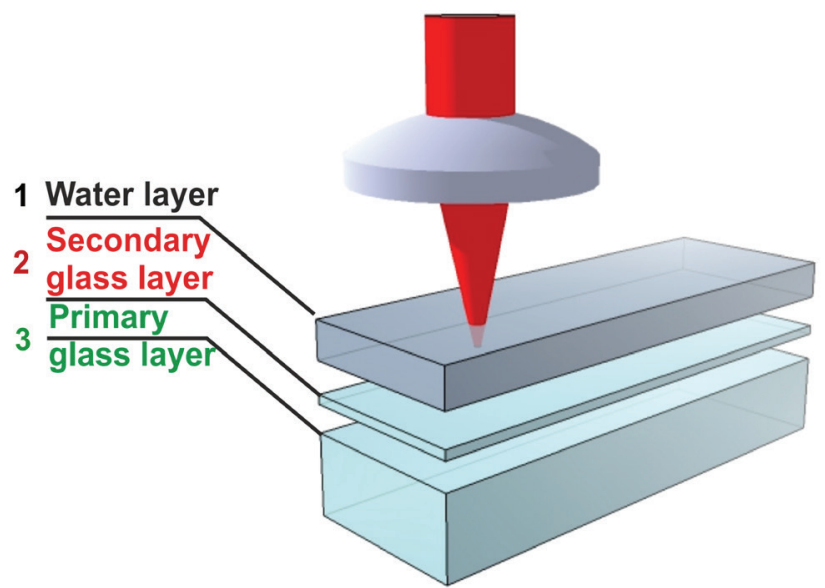

Fig. 3 Method for improving the quality of cuts by introducing a secondary glass layer (low thickness) on the base layer (primary)

that the quality of the fabricated complex shape object depends on the quality of a single straight cut. In fact, straight cut fabrication is a good way for determining the fabrication quality of the system. A top and side (profile) view image of the fabricated straight groove (with ot the secondary glass layer) is presented in Fig. 4. The top left image is acquired by a bright field microscope and reveals microcracks formed in the bulk, the bottom left SEM image reveals chipping of the edges (bulk microcracks are not visible). Conicity is visible on the right side of the image (profile). The profile view reveals the stated microcracks as well. Again, such an outcome is most likely due to diffraction and refractions of the impinging beam at the formed edges of the groove. However these effects take the biggest toll in terms of quality in the upper part of the sample $(\sim 200 \mu \mathrm{m}$ from the top surface of the sample) whereas the lower part is relatively undamaged. Therefore

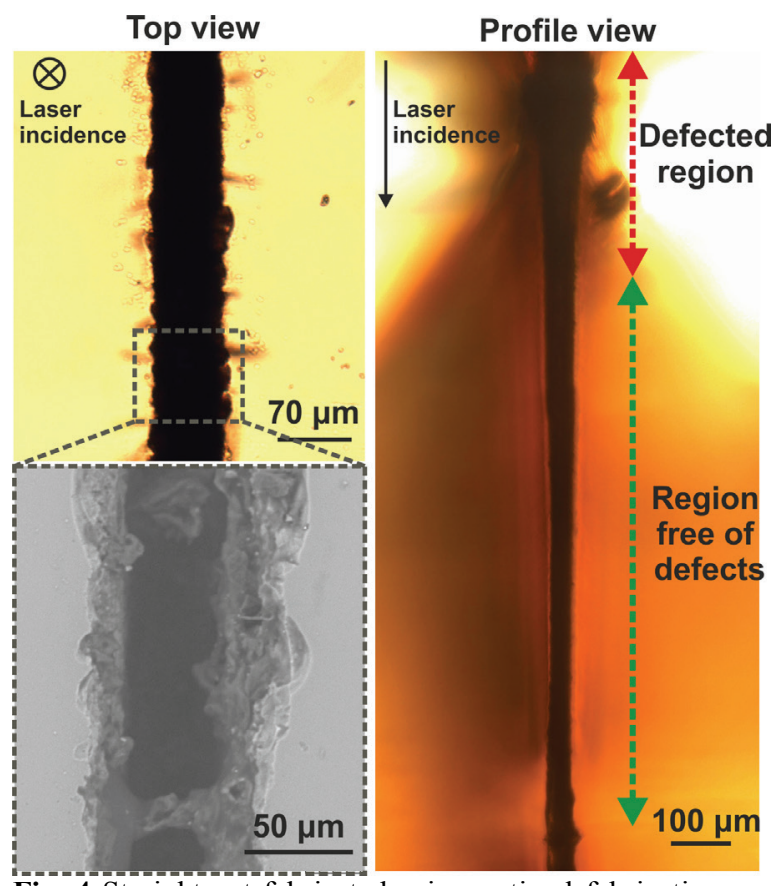

Fig. 4 Straight cut fabricated using optimal fabrication parameters (see text). The left part of the image shows a top view of the groove, the right part demonstrates the profile view 
Without secondary glass layer

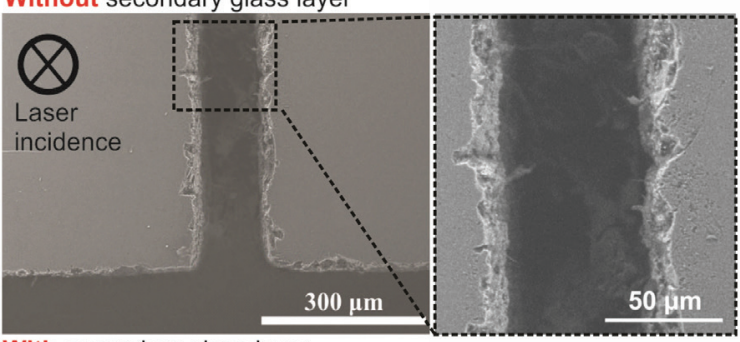

With secondary glass layer

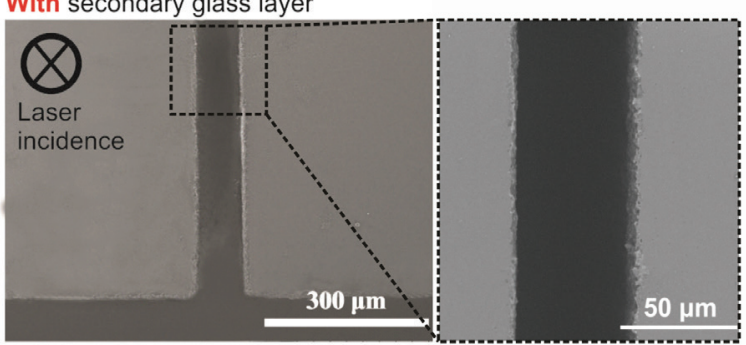

Fig. 5 Straight cuts fabricated using optimal fabrication parameters (see text). The top part of the image demonstrates a typical fabrication outcome when the secondary glass layer is absent. The bottom part shows improved quality when the additional glass layer is used.

the main reason for putting the secondary glass layer on top of the base layer (Fig. 3) is containment of this defected region in the secondary glass layer. After fabrication the secondary glass layer is discarded and all that is left is a cut possessing fewer imperfections.

It is worth mentioning that simply putting the secondary glass layer on top of the primary layer will not yield better result. In order to achieve increased quality one must apply strong pressure (the force applied depends on the materials used, their dimensions and the shape of the samples) to bring the two glass samples as close together as possible. A typical gap between the two glass samples that would yield better results in terms of quality is approximately $\lambda / 4$ (the gap was estimated by observing Newton rings when pressure is applied to the sample). An increase in observed cut quality when the gap between the samples is $\lambda / 4$ is due to several reasons. First, as mentioned above, due to reflection, diffraction, refraction the resulting interference pattern on the walls and on the bottom glass slide is different and a smaller gap results in less energy absorbed at the bottom sample's surface where the damage threshold is lower than the bulk. Second, when ablation of the sample occurs strong shock waves propagate in all directions, this leads to an even further increase in the gap between the glass samples and as result the sample is being ablated as if virtually no primary glass layer is present. Third, a relatively large gap between the glass slides results in better plasma-target coupling near the edges of the ablated groove which further decreases the quality of the cut [28].

An even better approach is forming an optical contact between the two glass samples. This makes the fabrication relatively easy and the entire setup is exactly the same as described in section 2. A comparison of cut grooves with and without the secondary glass layer is presented in Fig. 5. Here the improvement is clearly visible. Chipping (size of broken of chunks of glass) was reduced from $\sim 10-15$ $\mu \mathrm{m}$ to $\sim 0.5-3 \mu \mathrm{m}$. This decrease of chipplet size corresponds to a reduction of $\sim 600 \%$. The hole entrance-exit

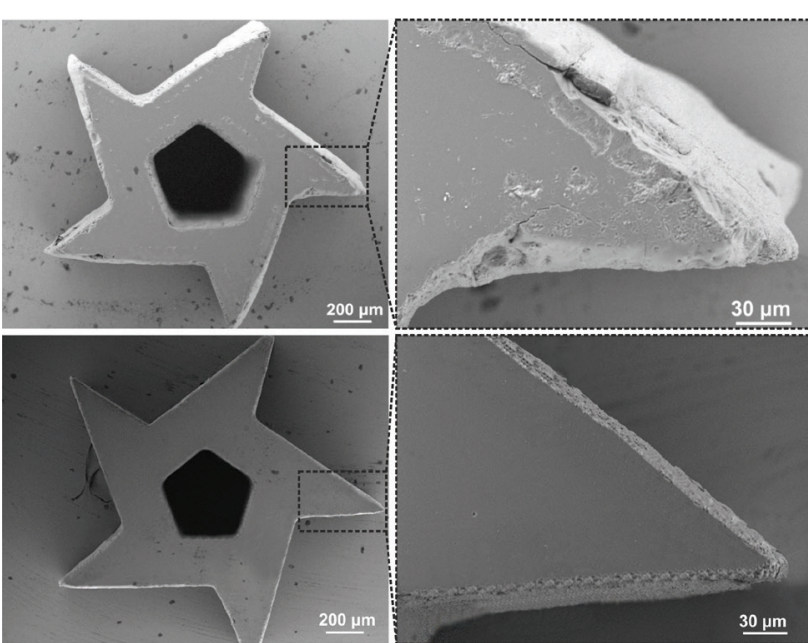

Fig. 6 Comparison of complex shape object fabrication when the secondary glass layer is absent (top) ant when it is implemented (bottom)

ratio was reduced from $\sim 3$ to $\sim 1.2-1.5$. These values correspond to reduction of $\sim 250 \%$. In addition, cracks that were formed in the bulk (visible in Fig. 4 right side) are totally eliminated. The roughness of the cut was estimated using a profilometer and equalled 4 - $5 \mu \mathrm{m}$ regardless if the secondary glass layer was used or not. A comparison on complex shape fabrication is depicted in Fig. 6. The better quality shape is a result of the percentile decrease in the imperfections that occur in the fabrication process. Overall it can be noted that better quality of complex shape objects fabricated with our proposed setup is easily implemented. Due to the added secondary glass layer the fabrication rate slightly decreases however we report only a decrease of approximately $15 \%$ in the fabrication rate (fabrication of the objects shown in Fig. 6 took: $\sim 3$ minutes (top) and 3.5 minutes (bottom)). Such a method with improved quality of cuts could be used for rapid fabrication of micromechanical components for various applications. The added secondary glass layer even though slightly decreases the fabrication rate does not add additional complexity to the fabrication system thus implementation of such a method is worth the effort if higher quality of processed samples is desired.

\section{Conclusion}

A fabrication system has been built for rapid cutting of holes and other complex-shaped objects in soda-lime glass with high intensity femtosecond pulse generated filaments. Direct ablation of glass samples with laser filaments can be realised by introducing a thin water layer above the samples. Such a water layer due to several competing nonlinear phenomena eliminates the need for precise positioning and translation along the $\mathrm{Z}$ axis. In addition, introducing a secondary (low thickness) glass layer on top of the base layer and keeping the fabrication system intact yields far better results in terms of quality while the decrease in fabrication rate is minimal. We demonstrated that with such a technique it is possible to drill holes and complex shape objects having perimeters greater than several $\mathrm{mm}$ in a matter of a few minutes. 


\section{Acknowledgments}

The authors thank the European Social Fund Agency for the support according to research grant No. VP1-3.1-SMM10-V-02-007 (Development and utilization of new generation industrial laser material processing using ultrashort pulse lasers for industrial applications).

\section{References}

[1] Ch. B. Schaffer, A. Brodeur and E. Mazur: Meas. Sci. Technol., 12, (2001) 1784.

[2] A. Ran, L. Yan, D. Yan-Ping, F. Ying, Y. Hong and G. Qi-Huang: Chinese Phys. Lett., 21, (2004) 2465.

[3] Z. Wu, H. Jiang, Q. Sun, H. Guo, H. Yang and Q. Gong: Appl. Opt., 6, (2004) 671.

[4] R.L. Byer, A. Harkin, J. Ashmore, H.W. Stone, M. Shen and E. Mazur: Appl. Phys. Lett., 83, (2003) 3030.

[5] Ch. B. Schaffer, A. Brodeur, J. F. Garcia, and E. Mazur: Opt. Lett., 26, (2001) 2.

[6] D. Ye, Y. Guang-Jun, W. Guo-Rui, M. HongLiang, Y. Xiao-Na, and M. Hong: Chin. Phys. B., 21, (2012) 201.

[7] D. N. Vitek, D. E. Adams, A. Johnson, Ph. S. Tsai, S. Backus, Ch. G. Durfee, D. Kleinfeld, and J. A. Squier: Opt. Expr., 18(17), (2010) 18086.

[8] D. N. Vitek, E. Block, Y. Bellouard, D. E. Adams, S. Backus, D. Kleinfeld, Ch. G. Durfee and J. A. Squier: Opt. Expr., 18(24), (2010) 24673.

[9] E.Block, M. Greco, D. Vitek, O. Masihzadeh, D. A. Ammar, M. Y. Kahook, N. Mandava, Ch. Durfee and J. Squier: Opt. Expr., 4(6), (2013) 831.

[10] Y. Bellouard, A. Said, M. Dugan and Ph. Bado: Opt. Exp., 17, (2004) 2120.

[11] J. Gottmann, M. Hermans, and J. Ortmann: J. of Las. Micro / Nanoengineering., 8, (2013) 15.

[12] D. Wortmann, J. Gottmann, N. Brandt, and H. Horn-Solle: Opt. Exp., 16, (2008) 1517.

[13] Y. Bellouard, A. Champion, B. Lenssen, M. Matteucci, A. Schaap, M. Beresna, C. Corbari, M. Gecevičius, P. Kazansky, O. Chappuis, M. Kral, R. Clavel, F. Barrot, J. Breguet, Y. Mabillard, S. Bottinelli, M. Hopper, C. Hoenninger, E. Mottay and J. Lopez: J. of Las. Micro/Nanoengineering., 7, (2012) 1.
[14] S. Kiyama, S. Matsuo, S. Hashimoto and Y. Morihira: J. Phys. Chem. C, 113, (2009) 11560.

[15] A. Marcinkevičius, S. Juodkazis, M. Watanabe, M. Miwa, S. Matsuo, H. Misawa and J. Nishii: Opt. Lett., 26(5), (2001) 277.

[16] E. Gua, C.W Jeona, H.W. Choia, G. Ricea, M.D. Dawsona, E.K. Illyb and M.R.H. Knowlesb: Proc. of Symposium $\mathrm{H}$ on Photonic Processing of Surfaces, Thin Films and Devices, Strasbourg, (2003) p.462

[17] Y. Izawa, S. Tanaka, H. Kikuchi, Y. Tsurumi, N. Miyanaga, M. Esashi, M. Fujita: Proc. of 21 IEEE International Conference on Micro Electro Mechanical Systems, Tucson (2008) p.1084.

[18] D. P. Banks, K. S. Kaur, Ch. Grivas, C. Sones, P. Gangopadhyay, Ch. Ying, J. Mills, S. Mailis, I. Zergioti, R. Fardel, M. Nagel, T. Lippert, X. Xu, S. P. Banks and R. W. Eason: Proc. LAMP2009 - 5th Int. Congress on Laser Advanced Materials Processing, Kobe, (2009) p. 206.

[19] D.J. Hwang, T.Y. Choi and C.P. Grigoropoulos: J. Appl. Phys., 79, (2004) 605.

[20] S. Nikumb, Q. Chen, C. Li, H. Reshef, H.Y. Zheng, H. Qiu, D. Low: Thi. Sol. Films, 477 (1), (2005) 216.

[21]U. Eppelt, S. Russ, C. Hartmann, M. Sun, C. Siebert, W. Schul: Proc of 31st International Congress on Applications of Laser \& ElectroOptics, Anheim (2012) p. 105

[22] S. Butkus, E. Gaižauskas, D. Paipulas, Ž. Viburys, D. Kaškelytė, M. Barkauskas, A. Alesenkov, V. Sirutkaitis: Appl. Phys. A, 114(1), (2014) 81.

[23] V. Kudriašov, E. Gaižauskas and V. Sirutkaitis: Appl. Phys., 93, (2008) 571.

[24] S. Butkus, D. Paipulas, R. Sirutkaitis, E. Gaižauskas and V. Sirutkaitis: J. of Las. Micro/Nanoengineering 9(3), (2014) 213.

[25] C. Ohl, M. Arora, D. Dijkink, V. Janve and D. Lohse: Appl. Phys. Lett., 89, (2006) 074102.

[26]Z. Zhi-Yuan, Z. Yi, Z. Wei-Gong, L. Xin, L. Yu Tong, Z. Jie: Chin. Phys. Lett., 24(2), (2007) 501.

[27] S. Zhu, Y. F. Lu, and M. H. Hong: Appl. Phys. Lett., 79, (2001) 1396.

[28] P. Kongsuwan and Y. L. Yao: Proc. of The 29th International Congress on Applications of Lasers \& Electro-Optics , Anaheim (2010) p.1069

(Received: July 28, 2014, Accepted: December 19, 2014) 